\title{
Qualitative changes in phase-response curve and synchronization at the saddle-node-loop bifurcation
}

\author{
Janina Hesse, ${ }^{*}$ Jan-Hendrik Schleimer, ${ }^{\dagger}$ and Susanne Schreiber ${ }^{\ddagger}$ \\ Institute for Theoretical Biology, Department of Biology, Humboldt-Universität zu Berlin, \\ Philippstrasse 13, Haus 4, 10115 Berlin, Germany \\ and Bernstein Center for Computational Neuroscience Berlin, Berlin, Germany
}

(Received 24 June 2016; revised manuscript received 12 November 2016; published 3 May 2017)

\begin{abstract}
Prominent changes in neuronal dynamics have previously been attributed to a specific switch in onset bifurcation, the Bogdanov-Takens (BT) point. This study unveils another, relevant and so far underestimated transition point: the saddle-node-loop bifurcation, which can be reached by several parameters, including capacitance, leak conductance, and temperature. This bifurcation turns out to induce even more drastic changes in synchronization than the BT transition. This result arises from a direct effect of the saddle-node-loop bifurcation on the limit cycle and hence spike dynamics. In contrast, the BT bifurcation exerts its immediate influence upon the subthreshold dynamics and hence only indirectly relates to spiking. We specifically demonstrate that the saddle-node-loop bifurcation (i) ubiquitously occurs in planar neuron models with a saddle node on invariant cycle onset bifurcation, and (ii) results in a symmetry breaking of the system's phase-response curve. The latter entails an increase in synchronization range in pulse-coupled oscillators, such as neurons. The derived bifurcation structure is of interest in any system for which a relaxation limit is admissible, such as Josephson junctions and chemical oscillators.
\end{abstract}

DOI: 10.1103/PhysRevE.95.052203

\section{INTRODUCTION}

Different states of macroscopic network dynamics are a hallmark of complex systems such as the brain. In nervous systems, transitions between dynamical states constitute important switch points. For example, the emergence of socalled frustrated synchronization states (i.e., high-entropic, multistable network states verging between order and disorder) is thought to play a role in neural function and its pathologies [1]. Such transitions can, on the one hand, emerge as a consequence of the network topology, as it is found in the human connectome [2]. Here, we present a general case where a specific variation in single-neuron properties can drastically switch network synchronization properties, provided the cells' parameters are close to a critical transition point: the saddle-node-loop (SNL) bifurcation.

While this bifurcation is not unknown [3], our results demonstrate that its substantial, qualitative consequences for neural dynamics have so far not been sufficiently acknowledged. Moreover, we show that the SNL bifurcation is a ubiquitous feature in (planar) type I neuron models. Because a vast proportion of models [4-6] belongs to this class (describing neurons ranging from isolated gastropod somata [7] to hippocampal neurons [8,9]), this transition point and its implication need to be taken into account for biological function.

\footnotetext{
*janina.hesse@bccn-berlin.de

$\dagger$ jh.schleimer@hu-berlin.de

${ }^{\ddagger}$ www.neuron-science.de
}

Published by the American Physical Society under the terms of the Creative Commons Attribution 4.0 International license. Further distribution of this work must maintain attribution to the author(s) and the published article's title, journal citation, and DOI.
Different types of spike generation were first classified by Hodgkin [10] and later linked to particular bifurcations ruling the transition from rest to spiking [11,12]. Recently, the ability of neurons to change the mechanism of spike generation under physiological conditions has attracted the interest of both theoreticians and experimentalists [13-16]. Attention was mostly directed at the transition between the two traditional excitability types, which involve either a fold (saddle node) or a Hopf bifurcation [Fig. 1(a)], along with their differential subthreshold filtering properties [17-20]. Here, we investigate an alternative transition, which switches the spike onset from a saddle node on an invariant cycle (SNIC) bifurcation to a saddle homoclinic orbit (HOM) bifurcation [Fig. 1(b)]. This transition is organized by a codimension-two bifurcation: the SNL bifurcation [21,22]. As we demonstrate, the SNL bifurcation causes an abrupt change in the phaseresponse curve, with far-reaching functional consequences. For example, the increased ability of individual cells to form antiphase synchronization observed at an SNL bifurcation affects the dynamics of networks (Fig. 2), with potential relevance for various pathological conditions ranging from epilepsy to Parkinson's disease [25,26].

SNL bifurcations can occur with several bifurcation parameters, including the time constant of the gating kinetics [23]. In this study, we identify the separation of time scales between voltage and gating dynamics as the decisive bifurcation parameter, underlying the effect of other parameters, such as capacitance or temperature. Starting at a SNIC bifurcation in planar general neuron models, we demonstrate that a variation in the separation of time scales provokes a generic sequence of firing onset bifurcations. Compared to other bifurcation studies, which rely on a local unfolding of a codimensionthree bifurcation [27,28], our approach proves the generic bifurcation structure including the appearance and ordering of codimension-two bifurcations on a global scale not restricted to local analysis. The composed bifurcation diagram hence 
(a) Destabilization of resting state:
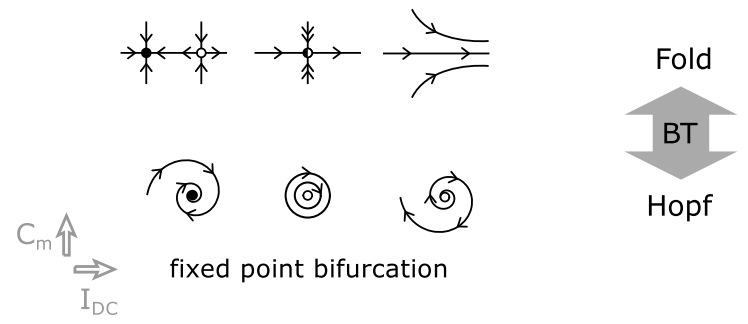

(b) Creation of stable limit cycle:

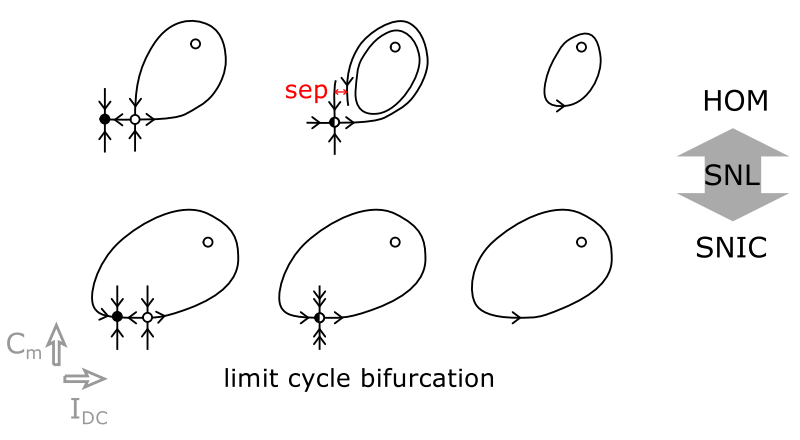

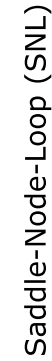

FIG. 1. The transition from rest to spiking in response to an increase in input current $I_{\mathrm{DC}}$ requires (a) that the resting state loses stability (illustrated are fold and subcritical Hopf bifurcations) and (b) the creation of a limit cycle [illustrated are saddle homoclinic orbit (HOM) and SNIC bifurcations]. The membrane capacitance $C_{\mathrm{m}}$ makes it possible to switch between these bifurcations. The separation function, sep, marked in red, measures the distance between the stable and unstable manifold of the saddle. The overlap of both, i.e., sep $=0$, results in a homoclinic orbit.

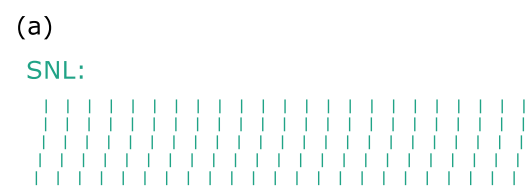

SNIC:

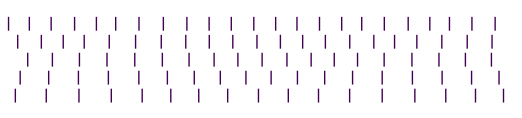

$0.5 \mathrm{~s}$
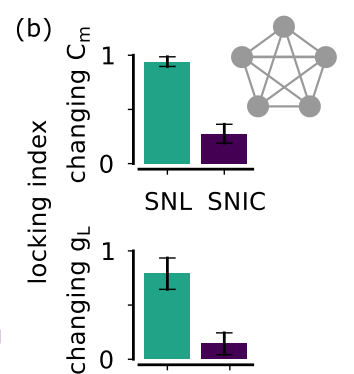

SNL SNIC
FIG. 2. (a) Spike raster plot of two small globally coupled networks of five Wang-Buzsaki models (see Appendix A), one close to the SNL bifurcation with $C_{\mathrm{m}}=1.47 \mu \mathrm{F} / \mathrm{cm}^{2}$, the other at a SNIC bifurcation with $C_{\mathrm{m}}=1 \mu \mathrm{F} / \mathrm{cm}^{2}$. Synaptic connections are modeled as voltage perturbations of $\varepsilon=0.39 \mathrm{mV}$. The frequency detuning of the neurons is approximately equally spaced between 5 and $11 \mathrm{~Hz}$. (b) Phase-locking index, calculated between pairwise neurons $i$ and $j$ with phase $\phi_{i, j}$ as $\left\langle e^{i 2 \pi\left(\phi_{i}-\phi_{j}\right)}\right\rangle$, where brackets denote temporal averaging. Error bars denote standard deviations. (Top) Locking index for the network when the capacitance is changed [as in panel (a)]. (Bottom) Locking index for the network when the leak conductance $g_{\mathrm{L}}$ is changed (instead of capacitance). $\varepsilon=0.08 \mathrm{mV}$, $C_{\mathrm{m}}=1 \mu \mathrm{F} / \mathrm{cm}^{2}$, and SNIC at $g_{\mathrm{L}}=0.1 \mathrm{mS} / \mathrm{cm}^{2}$ and SNL at $g_{\mathrm{L}} \approx 0.57 \mathrm{mS} / \mathrm{cm}^{2}$; all other parameters are identical to those in the top panel. Locking at both SNL bifurcations exceeds locking at the corresponding SNIC bifurcation. predicts the behavior of a class of neurons over the whole range of time-scale parameters and thereby warrants a direct comparison with biological neurons.

The organization of this article is as follows. Section II describes how the phase-response curve can be identified from the limit cycle of a dynamical system. With this relation established, Sec. III proves that a symmetry breaking of the phase-response curve occurs at SNL bifurcations. The functional consequences for synchronization in spiking systems are discussed in Sec. IV. The significance of these consequences is perpetuated by the results in Sec. V, where we prove that SNL bifurcations generically occur in planar neuron models.

\section{CONDUCTANCE-BASED NEURON MODEL AND PHASE REDUCTION}

To investigate spike-based synchronization, our detailed, conductance-based model neurons are reduced to a phase description. The latter assumes tonic responses of a meandriven neuron [18]; i.e., spikes are emitted with a mean spike rate, $f$, in response to a constant mean intensity, $I_{\mathrm{DC}}$, and their occurrence is modulated by inputs sufficiently weak to only shift spike times $\left\{t_{k}^{\mathrm{sp}}\right\}$. The spike train is $y(t)=\sum_{k} \delta\left(t_{k}^{\mathrm{sp}}-t\right)$.

The dynamics of the membrane voltage $v$ follows a current balance equation, $I_{\mathrm{DC}}=I_{\text {cap }}+I_{\text {ion }}$. The input equals the capacitive current, $I_{\text {cap }}=\frac{d C_{\mathrm{m}} v}{d t}$ (with membrane capacitance $C_{\mathrm{m}}$ ) and an ionic current, $I_{\text {ion }}=I_{\text {ion }}\left(v, m_{i}, \ldots\right)$, which is a function of $v$ itself and the open probability of ion channels given by their gating variables, $m_{i}$. Combined, this conductance-based neuron model forms a dynamical system, $\dot{X}=F(X)$, with the structure

$$
\left(\begin{array}{c}
\dot{v} \\
\dot{m}_{i} \\
\ldots
\end{array}\right)=\left(\begin{array}{c}
\frac{1}{C_{\mathrm{m}}}\left[I_{\mathrm{DC}}-I_{\mathrm{ion}}\left(v, m_{i}, \ldots\right)\right] \\
\frac{m_{i}^{\infty}(v)-m_{i}}{\tau_{m_{i}}(v)} \\
\cdots
\end{array}\right),
$$

where the overdot denotes the derivative with respect to time and $F$ determines the dynamics of the unperturbed system. Synaptic inputs are modeled as instantaneous voltage perturbations: If a spike occurs at time $t^{\mathrm{sp}}$ in the presynaptic neuron, then $v_{\text {post }}\left(t_{\text {pre }}^{\mathrm{sp}}\right) \mapsto v_{\text {post }}\left(t_{\text {pre }}^{\mathrm{sp}}\right)+\varepsilon$, where $\varepsilon=$ $I_{\mathrm{syn}} C_{\mathrm{m}}^{-1} \int_{t^{\mathrm{sp}}-\Delta}^{t^{\mathrm{sp}}+\Delta} d r \delta\left(r-t^{\mathrm{sp}}\right)$ results from the integration of $\delta$ currents of amplitude $I_{\text {syn }}$. The dynamical variables consist of the voltage and the gating variables. The gating is typically modeled by first-order kinetics (for details, see Appendix A).

The input $I_{\mathrm{DC}}$ acts as bifurcation parameter for both the fixed-point destabilization and the limit-cycle creation (Fig. 1). For our analysis, we focus on neuron models in which the fixed point loses stability at a fold bifurcation.

The study of spike synchronization is facilitated by reducing the high-dimensional dynamics to a single phase equation. One way to formally obtain such a reduction from a biophysical model of membrane-voltage dynamics is to find the input-output (I/O) equivalent phase oscillator [29]. The mapping of input to spike times is given by the phase-response curve $(P R C)$ of the neuron [30]. The PRC, $Z$, relates the timing of the occurrence of a weak perturbation to the resulting temporal advance or delay of the following spike, $Z: \phi \mapsto \Delta \phi$. The spike times $\left\{t_{k}^{\mathrm{sp}}\right\}$ correspond to the level 
crossings of the phase, $\phi\left(t_{k}^{\mathrm{sp}}\right)=k$ for $k \in \mathbb{Z}$, so that the spike train can be written as $y[\phi(t)]=\sum_{k} \delta[\phi(t)-k]$. The occurrence of spikes in neuron $i$, if receiving inputs from another neuron $j$, is governed by the phase equation

$$
\dot{\phi}_{i}=f_{i}+Z\left(\phi_{i}\right) y\left(\phi_{j}\right)+\xi_{i}(t)
$$

The intrinsic noise $\xi_{i}(t)$ of each neuron is assumed to be a zero-mean white-noise process, $\left\langle\xi_{i}(0) \xi_{i}(\Delta t)\right\rangle=\sigma^{2} \delta(\Delta t)$.

In the following, the mean spike rate, $f$, in response to the mean drive, $I_{\mathrm{DC}}$, and the PRC, $Z(\phi)$, are implicitly taken to be functions of the parameters of the detailed neuron model introduced above in Eq. (1). The phase oscillator in Eq. (2) is then used throughout the paper to predict synchronization properties of neurons.

To identify the I/O equivalent phase model in Eq. (2), the PRC needs to be calculated for the conductance-based model in Eq. (1). From a dynamical systems perspective, the PRC $Z$ is the periodic solution to the adjoint of the first variational equation of the unperturbed dynamics in Eq. (1), $\dot{X}=F(X)$,

$$
\frac{d Z}{d \phi}(\phi)=-J^{\top}(\phi) Z(\phi),
$$

where $T$ denotes the matrix transpose and $J=\frac{\partial F}{\partial X}$ is the Jacobian evaluated on the limit cycle. To comply with Eq. (2), the PRC associated with voltage perturbations needs to be normalized as $Z(\phi) F(\phi)=f, \forall \phi$. The resulting relation between PRC and parameters of the conductance-based neuron model allows us to consider synchronization at different firing onset bifurcations. In the following, we use the dynamics on the homoclinic orbit to deduce PRC properties of the limit cycle that arises from the homoclinic orbit, and, for convenience, we refer to the limit cycle PRC as the PRC at the limit-cycle bifurcation (SNIC or SNL), i.e., $Z_{\mathrm{SNIC}}$ or $Z_{\mathrm{SNL}}$.

\section{A FLIP IN THE DYNAMICS ALTERS THE PRC SYMMETRY AT AN SNL BIFURCATION}

In a first step, we infer the PRC from the dynamics at firing onset bifurcations, in particular around the SNL bifurcation. As bifurcations imply in general qualitatively different dynamics [31], limit-cycle dynamics are expected to change at the switch in firing onset dynamics at the SNL bifurcation. However, what are the specific consequences for the PRC and hence the synchronization ability of neurons? To answer this question, we start by discussing changes in limit-cycle dynamics at the SNL bifurcation. We then show that this also alters the PRC in such a profound way that it has, in turn, drastic implications for the resulting synchronization ability discussed in Sec. IV.

\section{A. Orbit flip}

We consider models with classical type I excitability where the transition from rest to repetitive firing is marked by (i) the elimination of the resting state in a fold bifurcation and (ii) the existence of a limit cycle to which the dynamics relax instead. This limit cycle is born at a limit-cycle bifurcation, which is in type I neurons typically a SNIC bifurcation. At a codimension-two SNL bifurcation, the limit-cycle bifurcation switches between a SNIC and a HOM bifurcation [Fig. 1(b)]. (a) orbit

(b) PRC
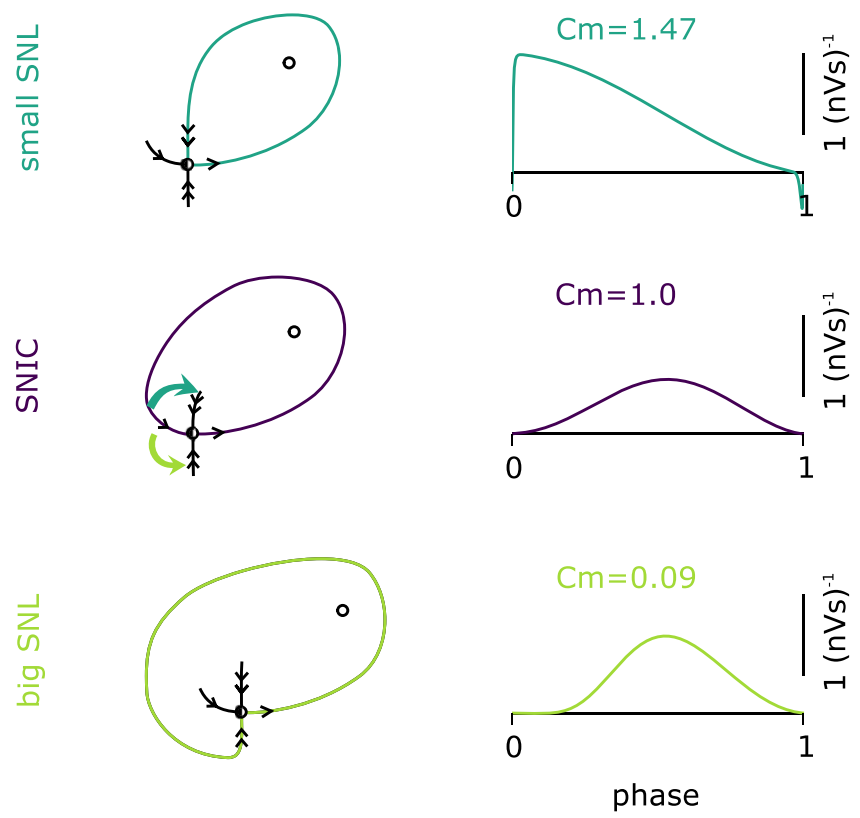

FIG. 3. (Top to bottom) (a) Schematic illustration of the orbits at small SNL bifurcation, nondegenerated SNIC bifurcation, and big SNL bifurcation, with semistable (small single arrow) and strongly stable manifold (double arrows). These bifurcations occur in the Wang-Buzsaki model for $I_{\mathrm{DC}} \approx 0.16 \mu \mathrm{A} / \mathrm{cm}^{2}, C_{\mathrm{m}} \approx$ $[1.47,1,0.09] \mu \mathrm{F} / \mathrm{cm}^{2}$. (b) The associated phase-response curves measured for $I_{\mathrm{DC}} 2 \%$ above the fold bifurcation.

The following, model-independent analysis focuses on the small SNL bifurcation that transitions from a SNIC orbit to a small HOM orbit [Fig. 3(a)], because it entails more drastic changes in PRC shape, as discussed later. The big SNL bifurcation (transitioning to a big HOM orbit) will be studied with numerical continuation (Sec. IV).

The limit cycle created at a HOM, SNIC, or SNL bifurcation arises from a homoclinic orbit to a saddle (HOM) or saddle node (SNIC, and also SNL). Under the assumption of sufficiently large limit-cycle periods, the slow velocity in the vicinity of these fixed points contracts the dynamics such that limit-cycle properties, e.g., period or PRC, can be extracted from a linear approximation around the fixed point.

The linearized dynamics around the saddle-node fixed point is given by its Jacobian. Assuming nondegeneracy, the Jacobian has a single zero eigenvalue, associated with the semistable manifold, while the other eigenvalues are strictly negative (strongly stable manifolds). Trajectories always leave the saddle node along the semistable manifold. When a trajectory loops around in a homoclinic orbit, it can either reapproach the saddle node along the same manifold (SNIC bifurcation) or along the much faster, strongly stable manifold (SNL bifurcation). The approach of the saddle node at an SNL bifurcation flips from the semistable manifold to one of the strongly stable manifolds (hence, orbit flip bifurcation [24]) [Fig. 3(a)]. For neuron models, this flip can be induced by a scaling of the relative speed in the voltage and gating kinetics (Fig. 4). When the saddle node disappears after the fold 
(a) SNIC bifurcation

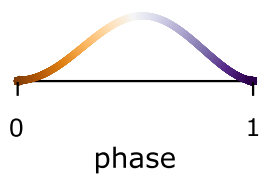

(b) SNL bifurcation
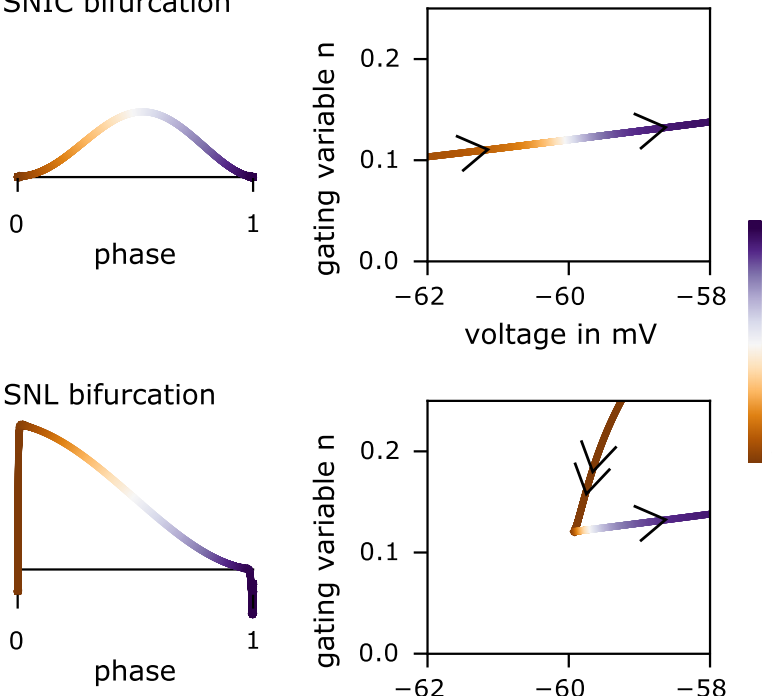

0
$\mathbb{0}$
$\frac{\pi}{0}$

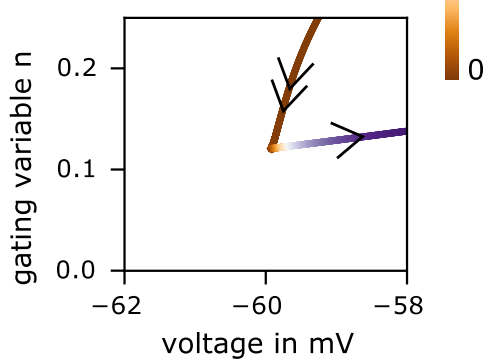

FIG. 4. Phase-response curve (left) and phase plot around the saddle node (right) for (a) a nondegenerated SNIC bifurcation with $C_{\mathrm{m}}=1 \mu \mathrm{F} / \mathrm{cm}^{2}$ and (b) a small SNL bifurcation with $C_{\mathrm{m}} \approx$ $1.47 \mu \mathrm{F} / \mathrm{cm}^{2}$ in the Wang-Buzsaki model with the limit-cycle period fixed in both cases to $2 \mathrm{~Hz}$.

bifurcation, its remaining ghost still dominates the resulting limit-cycle dynamics. The limit-cycle period drastically decreases around the SNL bifurcation (Fig. 5(a); see also [3]), mainly because of the separation of time scales between strongly stable and semistable manifold, which renders the approach along the strongly stable manifold much faster than the approach along the semistable manifold.

\section{B. PRC symmetry and Fourier modes}

Numerical continuation of several neuron models shows that the PRC is drastically altered at the SNL bifurcation. Exemplified in Fig. 3(b) for the Wang-Buzsaki model (Appendix A), the symmetric PRC at a (nondegenerated) SNIC bifurcation becomes increasingly asymmetric when an (a)

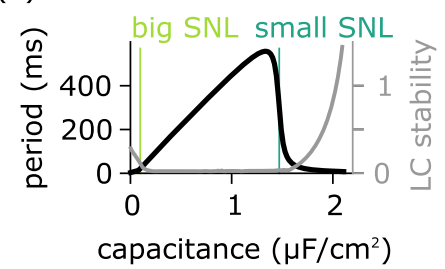

(b)

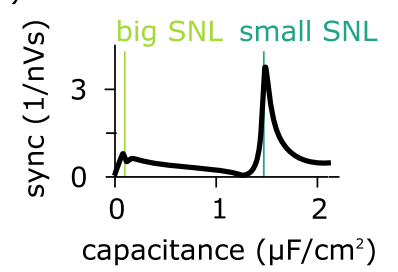

FIG. 5. Variation of the membrane capacitance $C_{\mathrm{m}}$ for the WangBuzsaki model with input fixed at $2 \%$ above limit-cycle onset at $I_{\mathrm{DC}} \approx 0.16 \mu \mathrm{A} / \mathrm{cm}^{2}$. (a) Limit-cycle period (black) and relative limit-cycle (LC) stability (gray) given by the ratio of the limit-cycle attraction time (inverse of the Floquet exponent) and the period. A small LC stability supports the validity of the phase description [36]. (b) Maximal amplitude of the odd part of the PRC, corresponding to the entrainment range when normalized by the coupling strength assuming $\delta$ coupling (abbreviated sync). increase in membrane capacitance tunes the model towards the SNL bifurcation. The strong asymmetry at the SNL bifurcation directly affects the synchronization ability of the neuron (see Sec. IV).

The sudden occurrence of PRC asymmetry at an SNL bifurcation can be directly inferred from the orbit flip in the dynamics described in the last section (Sec. III A). The PRC peaks when the phase reaches the ghost of the saddle node, where the slow dynamics allow infinitesimal perturbations to maximally advance phase. In the case of the SNIC bifurcation, the same velocity governs the approach and exit of the ghost, both aligned with the semistable manifold (Fig. 4; for details, see Appendix B). The orbit flip to the strongly stable manifold at the SNL bifurcation either decreases or increases the time spent on the approach compared to exit for the small or big SNL, respectively. This, in turn, breaks the symmetry of the PRC at the SNIC bifurcation by advancing or delaying the phase at which the maximum of the PRC resides.

Neglecting the fast approach at the small SNL bifurcation, it seems as if the flow of the limit-cycle trajectory is directly injected at the ghost. Because the exit dynamics at SNL and SNIC bifurcations are similar, the PRC at the small SNL bifurcation appears as a rescaled version of the second half of the PRC at the SNIC bifurcation, $Z_{\text {small SNL }}(\phi) \propto Z_{\text {SNIC }}(0.5 \phi+0.5)$. This reasoning is supported by numerical continuation [Figs. 3(b) and 4] and explains the observation that the limit-cycle period is approximately halved at the SNL bifurcation [Fig. 5(a)].

The necessity of the PRC symmetry breaking at the SNL bifurcation can also be seen from normal form theory. For the SNIC bifurcation (and the supercritical Hopf bifurcation), the PRC is a simple trigonometric function, $Z_{\mathrm{SNIC}}(\phi) \propto$ $1-\cos (2 \pi \phi)\left[Z_{\text {Hopf }}(\phi) \propto \sin (2 \pi \phi)\right][11,32,33]$. Approached from the SNIC, the small SNL bifurcation, however, registers a sudden emergence of higher Fourier modes in the PRC. On the other side of the small SNL bifurcation, the canonical PRC at a small HOM bifurcation is an exponential with some decay constant $\tau, Z_{\mathrm{HOM}}(\phi) \propto \exp (-\phi / \tau)[33,34]$. Hence, in contrast to the trigonometric PRCs with a single Fourier mode at the SNIC or supercritical Hopf bifurcations, the PRCs at HOM and small SNL bifurcations have an infinite amount of Fourier modes. This results in Gibb's phenomenon if finite approximations are used.

The significant increase in PRC Fourier modes, as well as the breaking in PRC symmetry, are generic properties of SNL bifurcations.

\section{SYNCHRONIZATION PEAKS AROUND SNL BIFURCATIONS}

The asymmetry of the PRC scales the frequency detuning over which a neuron entrains to its input (the width of the Arnold tongue $[35,36])$. The input can be either a periodic signal or the recurrent input from other neurons in a network. Here, we use synchronization in the sense of a constant phase relation between oscillators; compare Fig. 2. The relation between PRC and synchronization can be illustrated by two $\delta$-coupled phase oscillators, $\phi_{1,2}$, as defined in Eq. (2),

$$
\dot{\phi}_{1,2}=f_{1,2}+Q\left(\phi_{1,2}-\phi_{2,1}\right)+\xi_{1,2},
$$


where the coupling function $Q$ results from an averaging step if the interaction between both oscillators are assumed to be weak [37]. In the present pulse-coupled case this means

$$
Q(\Delta)=\int_{0}^{\infty} Z(\varphi) \delta(\varphi+\Delta) d \varphi=Z(\Delta) .
$$

The phase difference, $\psi=\phi_{1}-\phi_{2}$, evolves as $\dot{\psi}=\Delta f+$ $Q_{\text {odd }}(\psi)$, where $Q_{\text {odd }}(\psi)=Q(\psi)-Q(-\psi)$ is twice the odd part of the coupling function. Synchronization (i.e., a constant phase lag $\psi$ ) requires $\dot{\psi}=0$, and the maximal admissible frequency detuning $\Delta f=f_{1}-f_{2}$ is given by the image of $Q_{\text {odd }}$. In the case of $\delta$ coupling, $Q_{\text {odd }}$ is equal to twice the odd part of the PRC, $Z_{\text {odd }}$, so that phase locking occurs only if $\Delta f \in$ [min $Z_{\text {odd }}$, $\max Z_{\text {odd }}$ ]. In Fig. 5(b), the synchronization range $\max Z_{\text {odd }}-\min Z_{\text {odd }}$ is plotted. The increased synchronization range will also manifest itself in globally coupled networks of the type studied in Refs. [38,39]. For two coupled oscillators, a small SNL bifurcation favors alternated spiking, which is sometimes called antiphase synchronization. This is in contrast to the stable in-phase locking that is observed for PRCs shaped like a negative sine (see Supplemental Material in Ref. [27]).

The decisive factor for increased synchronization around the SNL points is the PRC symmetry breaking with the emergence of high-frequency Fourier modes, which govern the existence and stability of fixed points in the phase differences between oscillators. Of minor importance for the peak observed in Fig. 5(b) is the period reduction observed in Fig. 5(a), which counteracts the increase in synchronization in approach of the SNL points by scaling the PRC with the period. The network example from Fig. 2 also shows a significant change in synchronization when the period is held constant by a fixation of the mean firing rate. The PRC symmetry breaking with the emergence of high-frequency Fourier modes occurs generically at SNL bifurcations (Sec. III), such that the consequences derived in this section generalize to other oscillators beyond neuroscience. In particular, neurons close to an SNL bifurcation synchronize differently from what is expected for SNIC neurons that show traditional type I excitability.

\section{GENERIC OCCURRENCE OF SNL BIFURCATIONS}

The consequences of the SNL bifurcation discussed in Sec. IV will be of particular relevance for neuronal processing [40] if the SNL bifurcation generally occurs in realistic neuron models. Next, we demonstrate that indeed any two-dimensional, type I conductance-based neuron model can always be tuned to SNL bifurcations. More precisely, we show that the SNL bifurcation is an essential element in the bifurcation diagram that uses input current and membrane capacitance as control parameters. This bifurcation diagram also allows us to relate the SNL bifurcation to other bifurcations such as the Bogdanov-Takens (BT) bifurcation, classically termed the switch of type I/II excitability [37,41].

Concentrating on bifurcations relevant for neuronal spiking (i.e., bifurcations affecting a stable limit cycle), Fig. 6 shows a bifurcation diagram of the Wang-Buzsaki model (Appendix A) with input current and membrane capacitance as control parameters. Along the dimension spanned by the capacitance, two SNL bifurcations enclose the SNIC bifurcation. The lower SNL bifurcation corresponds to a big SNL bifurcation for which the arising limit cycle encircles the ghost of the
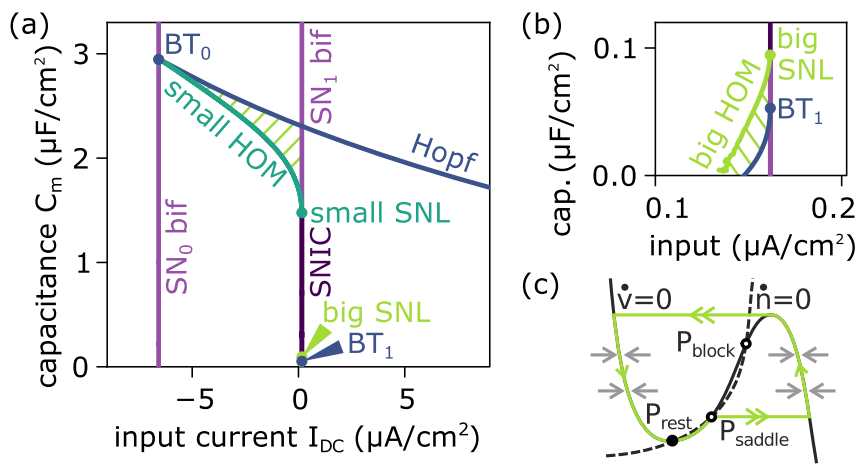

FIG. 6. (a) Bifurcation diagram of the Wang-Buzsaki model under variation of membrane capacitance $C_{\mathrm{m}}$ and input current $I_{\mathrm{DC}}$. With $C_{\mathrm{m}}=1 \mu \mathrm{F} / \mathrm{cm}^{2}$, the limit cycle arises from a SNIC bifurcation. Increasing $C_{\mathrm{m}}$ leads to the small SNL at $C_{\mathrm{m}} \approx 1.47 \mu \mathrm{F} / \mathrm{cm}^{2}$. Hatched areas mark bistability. (b) Decreasing $C_{\mathrm{m}}$ leads to the big SNL and then to a Bogdanov-Takens (BT) bifurcation. Note that a change of stability in the big HOM branch, not shown here, follows from Ref. [42]. (c) Schematic illustration for the limit $C_{\mathrm{m}} \rightarrow 0$, in which the system corresponds to a relaxation oscillator. Drawn in the state space of gating variable $n$ versus voltage $v$, the solid line with an inverted $\mathrm{N}$ shape represents the voltage nullcline, and the dashed line represents the gating nullcline. At some $I_{\mathrm{DC}}$, the resting state loses stability and a big HOM orbit around all fixed points (green) is created.

saddle node and the upper SNL bifurcation corresponds to a small SNL bifurcation for which the ghost of the saddle node lies outside of the limit cycle [Fig. 3(a)]. In particular, decreasing the capacitance, an SNL point is passed before the BT bifurcation is reached.

We show in Appendix $C$ that this bifurcation structure generalizes (under mild assumptions) to planar neuron models. The membrane capacitance $C_{\mathrm{m}}$ is used as a bifurcation parameter in the general bifurcation diagram that we construe, because it simply changes the time scale of the voltage dynamics [Eq. (1)]. The proof separately considers the lower and the upper parts of the bifurcation diagram. The lower part is based on the so-called relaxation limit with infinitely fast voltage dynamics that arises from the limit $C_{\mathrm{m}} \rightarrow 0$ [Fig. 6(c)], where the bifurcation structure is known [42]. Nonzero capacitance values are deduced from several observations that restrict the path of limit-cycle bifurcation branches in planar systems. The upper part of the bifurcation diagram is extracted from the unfolding of a BT point.

Our derivation may be an interesting starting point for similar results in other dynamical systems in which the time scale of a single dynamical variable is used as a bifurcation parameter. For our planar neuron models, we find that the SNIC bifurcation branch is generically enclosed by two SNL bifurcations that are reached by an adaptation of the voltage time scale. In particular, our results show that a continuous variation of the voltage time scale reaches the BT point only after passing one of the SNL bifurcations.

\section{DISCUSSION}

This article explores the intricate relation between SNL bifurcations, the changes in associated PRCs, and the resulting consequences for the ability of neurons to synchronize (Fig. 5). 
In a mean-driven regime [18] (i.e., in the presence of a stable limit cycle), drastic changes in neuronal processing in general [40], and synchronization in particular, can be expected if a bifurcation directly affects the stable limit cycle (and not only the fixed points). This is the case for the SNL bifurcation, with strong implications for synchronization, as we have shown here. In contrast, at a Bogdanov-Takens bifurcation, which is classically regarded as the transition point between type I and type II excitability, stable limit cycles are not directly involved. The BT-associated Hopf bifurcation in neuron models is typically subcritical, and the limit cycle arising at the subcritical Hopf bifurcation is unstable. Hence, the limit cycle changes bear only indirect relevance for mean-driven spiking. This provides an intuitive explanation for why the changes in synchronization we observed at the BT transition are minor compared to those of the saddlenode-loop bifurcation [Fig. 5(b)]. While the subcritical Hopf bifurcation can lead to substantial changes in subthreshold dynamics and filtering $[17,19,27,37,43]$, synchronization is modified only if the system behaves like a fluctuation-driven escape problem $[44,45]$, and not like the mean-driven regime considered here.

Moreover, we note that models in the vicinity of a BT point have a different bifurcation structure than the original Hodgkin-Huxley (HH) model [46]. In the HH model, the unstable limit cycle is born at a fold of limit cycles bifurcation (together with the stable limit cycle) and terminates in the subcritical Hopf bifurcation (destabilizing the fixed point). In contrast, in the normal form of the BT bifurcation, the unstable limit cycle is born at a HOM bifurcation [47]. This difference will probably affect the PRC of the stable limit cycle, for which the canonical shape is still unresolved [48]. Furthermore, the identified generic bifurcation sequence shows that a smooth change in time-scale parameters does not justify the previously used heuristic formula that exploits a single Fourier mode to interpolate between $Z_{\text {SNIC }}$ and $Z_{\text {Hopf }}[40,49,50]$.

As codimension-two bifurcation, the SNL bifurcation is reached in neuron models by an appropriate tuning of both the input current and one additional model parameter. Examples for the second control parameter are the membrane capacitance, maximal gating conductances, tonic inhibition [27], neuromodulators [14], or gating time constants [23]. With the membrane capacitance as a bifurcation parameter, we demonstrate for planar conductance-based models with a SNIC bifurcation that, ubiquitously, an SNL bifurcation is the first bifurcation reached for lowered or increased capacitance, respectively. With the three bifurcation parameterscapacitance, input, and leak conductance - the identified sequence of bifurcations collapses into a codimension-three cusp BT point $[27,28]$. This potentially generalizes the described bifurcation structure beyond the planar case.

Focusing on neuron models that spike at low firing rates, where the dynamics is dominated by the bifurcation that creates the limit cycle, allows us to draw model-independent conclusions. Furthermore, the phase description employed here demands for small inputs compared to the limit-cycle stability. The strong stability of the limit cycle around the SNL point [Fig. 5(a)] validates the phase reduction even for reasonably sized inputs. The low firing rates required for the center manifold reduction to be valid and the rel- atively weak synaptic connections are typical for cortical neurons $[4,5]$.

Our mathematical arguments (Appendix C) require one bifurcation parameter to be the relative time scale between state variables, which can take us to the relaxation limit. The membrane capacitance is one such parameter. The effective membrane capacitance depends on cell parameters, such as the morphology of the neuron or the myelination of its axon [51], and may hence be adapted on developmental or evolutionary time scales. Recent studies report reduced capacitance in human neurons [52,53], potentially due to the lipid composition of the membrane. While these studies consider mainly passive membrane properties, our work extends beyond and reports the implications of changed capacitance for spike dynamics. In contrast to the general assumption that the membrane capacitance is constant across all neurons, the reported variability in membrane capacitance suggests that evolution could directly tune the membrane capacitance to the proximity of an SNL point. Indeed, it seems that not only evolution, but also development acts on the membrane capacitance; for example, aging reduces $C_{\mathrm{m}}$ in rhesus monkeys [54].

Among the biologically relevant bifurcation parameters is the leak conductance $g_{\mathrm{L}}$, which affects the time-scale separation in a more indirect way than capacitance. Changes in leak can equally lead to an SNL bifurcation [27], with the accompanying increase in (antiphase) synchronization [Fig. 2(b)]. For example, the effective leak conductance can be changed by the amount of inhibition the neurons receives (shunting inhibition) [27] or by certain neuromodulators [55,56].

Based on our results, we predict that SNL points can be identified experimentally via the characteristic breaking of PRC symmetry. Experimental measurement of PRCs [27,57-61] can help to assess whether cellular dynamics are close to an SNL bifurcation. Moreover, our analysis suggests that a specific experimental technique-infrared neural stimulation-may require a careful interpretation. Specifically, infrared neural stimulation could not only excite neuronal tissue, but potentially also alter neuronal dynamics, because it has been shown to rely on a change in membrane capacitance, $C_{\mathrm{m}}$, to depolarize neurons and thus stimulate networks [62]. Hence, during infrared neural stimulation extended changes in capacitance could push the neurons closer to an SNL bifurcation, with consequences for neuronal dynamics and functionality exceeding a pure excitation.

Last but not least, our results provoke the question as to why neurons should, under natural conditions, favor a position close to an SNL point. On the one hand, the facilitation of antiphase synchronization around the SNL point may result in a frustrated network state with rich dynamical attractors for memory or information processing $[1,65]$. On the other hand, SNL bifurcations may also be relevant in pathology (e.g., epilepsy). Beyond neural networks [66], frustrated systems also underlie power blackouts [71], repressive gene networks [65], and social networks [72].

In summary, our study consists of two parts. First, we extracted the phase-response curve from the dynamics at an SNL bifurcation and used this knowledge to infer the associated synchronization abilities. Both the PRC asymmetry 
and its high Fourier modes are generic properties at SNL bifurcations. Thereby, our results generalize across neuron models, and are equally applicable to any system that allows for a phase reduction. Second, we have demonstrated that SNL bifurcations occur ubiquitously in a set of planar neuron models. With the time scale of one dynamical variable as bifurcation parameter, the structure of our proof is likely to extend to other systems with a subcritical Hopf bifurcation in the relaxation limit, such as lasers [73,74], Josephson junctions [75-78], and chemical reactions $[79,80]$. Together, both parts highlight the SNL bifurcation as a hitherto underestimated bifurcation with prominent importance for neuronal dynamics.

\section{ACKNOWLEDGMENTS}

We thank Martin Wechselberger for inspiring discussions and pointing out the connection to his work on the relaxation limit and Grigory Bordyugov for advice on numerical continuation with AUTO [81] and homoclinic bifurcations. Funded by the German Federal Ministry of Education and Research (Grants No. 01GQ0901 and No. 01GQ1403).

Authors J.H. and J-H.S. contributed equally to this work.

\section{APPENDIX A: GENERIC DEFINITION OF CONDUCTANCE-BASED NEURON MODELS}

We consider a generic class of conductance-based neuron models [27],

$$
\begin{gathered}
\dot{v}=\frac{I_{\text {cap }}(v, \ldots)}{C_{\mathrm{m}}}=\frac{1}{C_{\mathrm{m}}}\left[I_{\text {in }}-g_{\mathrm{L}}\left(v-v_{\mathrm{L}}\right)-I_{\text {gating }}\right], \\
I_{\text {gating }}=\sum_{i=0}^{n} g_{i}\left(v-v_{i}\right) \prod_{k=0}^{K} m_{i k}^{p_{i k}},
\end{gathered}
$$

where ion channel $i$ has maximal conductance $g_{i}$ and reversal potential $v_{i}$ and its open probability is given by a product of gating variables (potentially to some power of $p_{i k}$ ). Each gating variable $m_{i k}$ of ion channel $i$ is either a function of the voltage, $m_{i k}=m_{i k \infty}(v)$, or relaxes exponentially to its steady state value $m_{i k \infty}(v)$, with gating kinetics given by

$$
\dot{m_{i k}}=\frac{m_{i k \infty}(v)-m_{i k}}{\tau_{i k}(v)} .
$$

For numerical continuation, we use a single-compartmental version of the Wang-Buzsaki model for hippocampal pyramidal cells [9] with the dynamics

$$
\begin{aligned}
& \dot{v}=\left[I+g_{\mathrm{L}}\left(E_{\mathrm{L}}-v\right)+I_{\text {gate }}\right] / C_{\mathrm{m}}, \\
& \dot{h}=5\left[\alpha_{h}(v)(1-h)-\beta_{h}(v) h\right], \\
& \dot{n}=5\left[\alpha_{n}(v)(1-n)-\beta_{n}(v) n\right],
\end{aligned}
$$

with membrane capacitance $C_{\mathrm{m}}=1 \mu \mathrm{F} / \mathrm{cm}^{2}$; maximal conductances $\quad g_{\mathrm{L}}=0.1 \mu \mathrm{S} / \mathrm{cm}^{2}, \quad g_{\mathrm{Na}}=35 \mu \mathrm{S} / \mathrm{cm}^{2}$, $g_{\mathrm{K}}=9 \mu \mathrm{S} / \mathrm{cm}^{2} ;$ reversal potentials $E_{\mathrm{L}}=-65 \mathrm{mV}$, $E_{\mathrm{Na}}=55 \mathrm{mV}, \quad E_{\mathrm{K}}=-90 \mathrm{mV} ; \quad$ and the following functions:

$$
\begin{aligned}
I_{\text {gate }} & =g_{\mathrm{Na}} m_{\infty}(v)^{3} h\left(E_{\mathrm{Na}}-v\right)+g_{\mathrm{K}} n^{4}\left(E_{\mathrm{K}}-v\right), \\
m_{\infty}(v) & =\frac{\frac{v+35}{\exp [-0.1(v+35)]-1}}{\frac{v+35}{\exp [-0.1(v+35)]-1}-40 e^{-(v+60) / 18}} \\
\alpha_{h}(v) & =0.07 \exp [-(v+58) / 20] \\
\beta_{h}(v) & =\frac{1}{1+\exp [-0.1(v+28)]} \\
\alpha_{n}(v) & =-0.01 \frac{v+34}{\exp [-0.1(v+34)]-1} \\
\beta_{n}(v) & =0.125 \exp [-(v+44) / 80]
\end{aligned}
$$

\section{APPENDIX B: PRC SYMMETRY}

The PRC asymmetry at the SNL bifurcation is a direct consequence of the broken symmetry in the dynamics at the SNL bifurcation. This section gives more detail on the relationship between dynamics and PRC, both intuitively and with a mathematical argument. We will describe first how the dynamics at the SNIC bifurcation leads to a symmetric PRC and then show that these conditions are not met at the SNL bifurcation, predicting an asymmetric PRC at an SNL bifurcation. While the arguments are presented with a small SNL bifurcation in mind, they hold in a similar way for a big SNL bifurcation.

As introduced in the main text, the orbit at a SNIC bifurcation follows the semistable manifold of the saddle-node fixed point, which corresponds to the central manifold of a fold bifurcation. The zero eigenvalue of the Jacobian $J$ at the saddle node on the semistable manifold eliminates the linear term. The leading second-order term results in a parabolic normal form. For dynamics centered around $x=0$, stimulated with input $s$, the dynamics is

$$
\dot{x}=s+x^{2},
$$

where all variables are chosen unitless for convenience.

The dynamics is symmetric around the saddle-node fixed point; i.e., the orbit has corresponding velocities at the approach and exit of the saddle node. The orbit flip at the SNL bifurcation breaks this symmetry in the dynamics, and, as we will show, also in the PRC.

From a mathematical perspective, the normal form allows for a calculation of the PRC. We, however, will use the normal form to directly analyze PRC symmetry. For the SNIC bifurcation, the reflection symmetry of the PRC can be inferred from the symmetry of the dynamics: If $x(t)$ is a solution of the dynamical system given by Eq. (B1), then the same holds for $-x(-t) ; x(t)$ is hence point symmetric in time, $x(t)=-x(-t)$. Derivation of the right-hand side of Eq. (B1) by $x$ results in a Jacobian linear in $x$, which is hence point symmetric in $x, J(x)=-J(-x)$. Inserting both into the adjoint equation [Eq. (3)] directly leads to a $\mathrm{PRC}$ reflection symmetric in time, $Z_{\mathrm{SNIC}}(t)=Z_{\mathrm{SNIC}}(-t)$. In contrast, the asymmetric dynamics at the SNL bifurcation lead to an asymmetric PRC.

Intuitively, on an orbit that connects to a saddle-node fixed point, the dynamics becomes arbitrary slow at the fixed point. The limit cycle shows the slowest dynamics in the same region 
in state space, in proximity to the ghost of the former saddle node. A perturbation that propels the dynamics over the ghost of the saddle node will therefore maximally advance the next spike. The maximum of the PRC is at the phase value that corresponds to the saddle node. For a SNIC bifurcation, the PRC maximum lies at $\phi=0.5$ because the symmetric dynamics of a SNIC take equal time for the approach (from $\phi=0$ to $\phi=0.5$ ) and the exit (from $\phi=0.5$ to $\phi=1$ ) of the saddle node. In comparison, the PRC maximum is shifted towards the left at the SNL bifurcation, because the accelerated entry along the strongly stable manifold advances the saddle node to earlier phases. The shift of the maximum away from the center destroys the symmetric shape of the PRC.

The symmetry breaking generalizes beyond the SNL bifurcation: A saddle homoclinic orbit shows an asymmetric PRC [33] if the saddle has different stabilities along stable and unstable manifold and, hence, nonsymmetric dynamics. In summary, we showed that the symmetry breaking in the PRC is an immediate consequence of the symmetry breaking in the dynamics that occurs as orbit flip at the SNL bifurcation. Hence, the observed symmetry breaking in the PRC is a general property of the SNL bifurcation.

\section{APPENDIX C: MATHEMATICAL ARGUMENT FOR THE GENERIC OCCURRENCE OF THE SNL BIFURCATION IN PLANAR MODELS}

We show in the following that, with a variation of a time-scale parameter, such as capacitance, in a broad set of planar conductance-based models, a SNIC bifurcation is always enclosed by two SNL bifurcations and that a decrease in capacitance passes the big SNL bifurcation and only afterwards reaches the BT point. Beyond the BT point, a Hopf bifurcation destabilizes the resting state before the fold bifurcation occurs.

To this aim, we prove that the general structure of the bifurcation diagram (Fig. 6) holds for any planar neuron model that conforms with our assumptions stated below.

\section{Model definition}

We consider a generic class of type I planar conductancebased neuron models. The single gating variable, $n$, commonly models the opening and closing of a restorative current originating, say, from the potassium ion channel. The dynamics is given by

$$
\left(\begin{array}{c}
\dot{v} \\
\dot{n}
\end{array}\right)=F(v, n)=\left(\begin{array}{c}
\frac{1}{C_{\mathrm{m}}}\left(I_{\mathrm{DC}}-I_{\mathrm{ion}}\right) \\
\frac{n_{\infty}(v)-n}{\tau_{n}(v)}
\end{array}\right),
$$

with $\quad I_{\text {ion }}(v, n)=g_{\mathrm{L}}\left(v-v_{\mathrm{L}}\right)+g_{\mathrm{Na}} m_{\infty}(v)\left(v-v_{\mathrm{Na}}\right)+$ $g_{\mathrm{K}} n\left(v-v_{\mathrm{K}}\right)$; compare Eq. (A1).

We chose the model such that it fulfills the following assumptions.

(A1) The firing onset of the model occurs, for some capacitance value $C_{\mathrm{SNIC}}$ and a specific input current $I_{\mathrm{DC}}=I_{\mathrm{SN} 1}$ (the threshold current), at a nondegenerated SNIC bifurcation.

(A2) We demand that at the capacitance $C_{\text {SNIC }}$ the subthreshold dynamics for $I_{\mathrm{DC}}<I_{\mathrm{SN} 1}$ relax to a single stable fixed point, the resting state. We furthermore assume that with an increase in input current, the limit-cycle dynamics eventually terminates in a bifurcation denoted excitation block, after which the dynamics relaxes again to a stable fixed point. This assumption prevents diverging dynamics.

(A3) The nullcline of the voltage has an inverted $\mathrm{N}$ shape.

(A4) We require that $n_{\infty}(v)$ from Eq. (C1) is an increasing, positive, bounded, twice differentiable function that becomes sufficiently flat in the limit $v \rightarrow \pm \infty, \lim _{v \rightarrow \pm \infty} v \partial_{v} n_{\infty}(v)=0$. This assumption allows us to use results from Ref. [27].

All of these assumptions are fulfilled in common neuron models with type I excitability.

\section{Construction of the bifurcation diagram}

The following proof establishes an ordering in a sequence of limit-cycle bifurcations, whereby a SNIC is enclosed by two SNL bifurcations. The ordering is established by analyzing the relaxation limit as an anchoring point. We thereby capitalize on recent results from the relaxation limit, $C_{\mathrm{m}} \rightarrow 0$. As we will show, the ordering that arises in this limit along $I_{\mathrm{DC}}$ implies the same ordering along $C_{\mathrm{m}}$, mainly because limit-cycle bifurcation branches cannot cross in planar systems.

The limit-cycle bifurcation branches that we consider lie in the region with $I_{\mathrm{DC}} \leqslant I_{\mathrm{SN} 1}$, because, for neuronal firing, the limit-cycle creation has to happen before (i.e., at lower $\left.I_{\mathrm{DC}}\right)$ or at the fold bifurcation at which the resting state is eliminated. $C_{\mathrm{SNIC}}$ separates the region $I_{\mathrm{DC}} \leqslant I_{\mathrm{SN} 1}$ into a lower and an upper subregion. Since the occurrence of limit-cycle bifurcations at $C_{\mathrm{SNIC}}$ is prevented by the requirement (A2) that stable dynamics are given by a unique fixed point, all limit-cycle bifurcation branches lie either in one or the other subregion. In the proof, we start with the lower subregion and then consider the upper one.

\section{The lower part of the bifurcation diagram, $C_{\mathrm{m}}<C_{\mathrm{SNIC}}$}

Observation 0: Vertical fold bifurcation branches-Fixed point location depends on $I_{\mathrm{DC}}$, but not on $C_{m}$. The nullclines of Eq. (C1) are given by $I_{\mathrm{DC}}-I_{\text {ion }}(v, n)=0$ and $n=n_{\infty}(v)$. The nullclines are independent of $C_{\mathrm{m}}$, and therefore also the location of the fixed points, because the fixed points sit at intersections of the nullclines. Hence, the location of the fold bifurcations is also independent of $C_{\mathrm{m}}$, which ensures that the fold branches [marked with $\mathrm{SN}$ in Fig. 6(a)] are vertical in a bifurcation diagram of $C_{\mathrm{m}}$ versus $I_{\mathrm{DC}}$.

Based on the inverted $\mathrm{N}$ shape of the voltage nullcline and the monotonous shape of the gating nullcline, we can infer the existence of one to three fixed points. For the following discussion, we name these fixed points; a visualization of our nomenclature is shown in Fig. 6(c). The number and location of the fixed points is set by the input current $I_{\mathrm{DC}}$, which shifts the voltage nullcline up or down in the state space. For low, i.e., subthreshold $I_{\mathrm{DC}}$, the model has a single, stable fixed point, $P_{\text {rest }}$. With an increase in $I_{\mathrm{DC}}$, the knee of the voltage nullcline approaches the gating nullcline from below and results in a fold bifurcation at some $I_{\mathrm{DC}}=I_{\mathrm{SN} 0}$. The fold bifurcation creates a saddle, $P_{\text {saddle }}$, and a node, $P_{\text {block }}$. Our assumptions ensure that $P_{\text {block }}$ is unstable because (A2) requires that $P_{\text {rest }}$ is the only stable fixed point at $C_{\mathrm{m}}=C_{\mathrm{SNIC}}$. Increasing the input current further leads to a second fold bifurcation at some $I_{\mathrm{DC}}=I_{\mathrm{SN} 1}$. 
This fold bifurcation annihilates $P_{\text {rest }}$ and $P_{\text {saddle }}$. Beyond the bifurcation, $P_{\text {block }}$ remains as the only surviving fixed point.

The saddle fixed point $P_{\text {saddle }}$ exists only between $I_{\mathrm{SN} 0}$ and $I_{\mathrm{SN} 1}$. The association of HOM bifurcations with saddles directly constrains their bifurcation branches to the region $I_{\mathrm{SN} 0} \leqslant I_{\mathrm{DC}} \leqslant I_{\mathrm{SN} 1}$. In an analogous way, Hopf bifurcation branches are constrained by the existence of the associated focus fixed point: The Hopf branch that destabilizes the resting state $P_{\text {rest }}$ is restricted to input currents below $I_{\mathrm{SN} 1}$, and the other Hopf branch that changes the stability of $P_{\text {block }}$ is restricted input currents above $I_{\mathrm{SN} 0}$. Further constraints will be developed throughout the following arguments.

Observation 1: Starting points for the branches of big HOM and neighboring Hopf bifurcation-Anchoring the bifurcation diagram in the limit $C_{m} \rightarrow 0$ yields $I_{\text {big HOM }}<I_{\text {Hopf }}$. In the limit $C_{\mathrm{m}} \rightarrow 0$, the conductance-based model is transformed into a relaxation oscillator with voltage as the fast variable, as sketched in Fig. 6(c) [82]. For this limit, de Maesschalck and Wechselberger have identified the full bifurcation structure for generic planar neuron models [42]. Their Theorem 2 demonstrates for sufficiently small $C_{\mathrm{m}}$ that an increase in $I_{\mathrm{DC}}$ results for model neurons such as ours in a generic sequence of bifurcations. Relevant for our consideration is the occurrence of a big HOM bifurcation at input $I_{\text {big HOM }}$ and a subcritical Hopf bifurcation that destabilizes $P_{\text {rest }}$ at $I_{\text {Hopf }}$. Their full bifurcation structure ensures furthermore that neither the big HOM branch nor the Hopf branch returns to the limit $C_{\mathrm{m}} \rightarrow 0$, which is important to ensure the existence of a codimension-two bifurcation at the other end. They state an ordering of the bifurcation currents, $I_{\text {big HOM }}<I_{\mathrm{Hopf}}<I_{\mathrm{SN} 1}$, which will be used in the following to infer the same ordering at finite values of $C_{\mathrm{m}}$.

Lemma 1: HOM branches cannot "bend backwards"A variation in $C_{m}$ generically breaks homoclinic orbits to hyperbolic fixed points. In order to constrain the location of HOM bifurcations in subsequent paragraphs, we want to show that the tracing of a HOM branch leads us always in one direction along the input current (increasing or decreasing input). Equivalently, we can show that a HOM branch cannot "bend backwards" along the input current dimension. This is the case if we show that HOM branches cannot have "vertical parts": A HOM branch cannot align with a parameter variation exclusively in $C_{\mathrm{m}}$, because, as we show with this lemma, a variation in $C_{\mathrm{m}}$ generically breaks the homoclinic orbit.

Homoclinic orbits arise when the trajectory of the unstable direction of a fixed point connects to its stable direction, i.e., stable and unstable manifold overlap. A parameter variation can separate stable and unstable manifolds from each other, allowing for the definition of a distance. This distance is measured by the so-called separation function, sep [Fig. 1(b)]. For parameter values that lie on the HOM branch, the separation function is zero, $\operatorname{sep}\left(C_{\mathrm{HOM}}\right)=0$, and becomes nonzero, $\operatorname{sep}\left(C_{\mathrm{m}}\right) \neq 0$, if a variation in the parameter breaks the homoclinic orbit, i.e., leaves the HOM branch. This is analogous to a nonzero value of the partial derivative of the separation function, which is known as the Melnikov integral, $M$ [for a derivation in planar systems, see, for example, Ref. [83], leading to Eq. (6.12), which we use in Eq. (C2)].

A variation in $C_{\mathrm{m}}$ breaks the homoclinic orbit if the corresponding Melnikov integral evaluated on the homoclinic orbit is nonzero [24]. The Melnikov integral with respect to $C_{\mathrm{m}}$ for a homoclinic orbit with flow $h(t)$ is

$$
\begin{aligned}
M & =\int_{-\infty}^{\infty} K(t) F(h(t)) \frac{\partial F(h(t))}{\partial C_{\mathrm{m}}} d t \\
& =-\int_{-\infty}^{\infty} K(t) \frac{\left[I_{\mathrm{DC}}-I_{\mathrm{ion}}(h(t))\right]^{2}}{C_{\mathrm{m}}^{3}} d t,
\end{aligned}
$$

where $K(t)=\exp \left[-\int_{0}^{t} \operatorname{div} F(h(s)) d s\right]$. For our system, the Melnikov integral is strictly positive, $0<M$, because (i) $K(t)$ is, as an exponential function, strictly positive, $\forall t: 0<K(t)$, and (ii), because we implicitly assume the existence of a homoclinic orbit, the difference of ionic and injected currents cannot be zero at all times; hence, $\exists t:\left[I_{\mathrm{DC}}-I_{\text {ion }}(h(t))\right]^{2}>0$. With that, the capacitance breaks the homoclinic orbit, and thus tracing a HOM branch along one direction results either in continuously increasing or decreasing input current values on the branch. This lemma is used in the following Observation 2 in order to pursue the big HOM branch starting in the limit $C_{\mathrm{m}} \rightarrow 0$ (see Observation 1 ).

Observation 2: The big HOM branch eventually approaches the fold bifurcation at $I_{\mathrm{SN} 1}$. Based on the directionality of the big HOM branch derived in the literature, we will show in this observation that the big HOM branch eventually approaches the fold bifurcation branch at which the resting state collides with the saddle. The point of contact corresponds to an SNL bifurcation, as we will show in subsequent paragraphs.

The statement of Theorem 2 by de Maesschalck and Wechselberger states for sufficiently small $C_{\mathrm{m}}$, in addition to the ordering used in Observation 1, that the big HOM branch departs from its starting point to the right, i.e., in the direction of increasing input current [42]. This directionality of the big HOM branch generalizes to larger values of $C_{\mathrm{m}}$, because Lemma 1 prevents "backward bends" of HOM branches. Given that the big HOM branch does not return to the limit $C_{\mathrm{m}} \rightarrow 0$ (Observation 1), the big HOM branch eventually has to approach the fold bifurcation at $I_{\mathrm{SN} 1}$. The next lemma ensures that the connection point is an SNL point.

Lemma 2: A HOM branch and the fold branch at $I_{S N 1}$ connect in an SNL bifurcation-A HOM branch is stable when it connects to a nondegenerated fold bifurcation involving a stable node. An SNL bifurcation involves a stable homoclinic orbit that transitions between a HOM bifurcation and a SNIC bifurcation. The homoclinic orbit of the HOM branch is stable if the associated saddle quantity is negative (the sum of the two eigenvalues of the associated fixed point). At the connection point with the fold branch, the homoclinic orbit is associated with a saddle-node fixed point arising from the collision of a stable node and a saddle. It has one zero eigenvalue (prerequisite for the fold bifurcation) and one negative eigenvalue (the former stable node sets the stability of the strongly stable manifold). The sum evaluates to a negative saddle quantity, ensuring a stable homoclinic orbit, and hence an SNL bifurcation.

Lemma 3: The bifurcation sequence in the lower part of the bifurcation diagram-For $I_{\mathrm{DC}}=I_{\mathrm{SN} 1}$, increasing $C_{m}$ from zero passes first a BT point, then an SNL point, before a nondegenerated SNIC bifurcation occurs, $C_{\mathrm{BT} 1}<C_{\mathrm{big} \mathrm{SNL}}<$ $C_{\text {SNIC }}$. Combining Observation 2 and Lemma 2, we conclude that the big HOM branch connects to the fold bifurcation 
branch at $I_{\mathrm{SN} 1}$ with a stable homoclinic orbit, i.e., in an SNL bifurcation. This big SNL bifurcation happens at some point $\left(I_{\mathrm{SN} 1}, C_{\text {big SNL }}\right)$, with $C_{\text {big SNL }}<C_{\mathrm{SNIC}}$ because the big HOM branch cannot pass the capacitance value of $C_{\text {SNIC }}$ as (A2) prohibits stable limit-cycle bifurcations for $I_{\mathrm{DC}}<I_{\mathrm{SN} 1}$. From Observation 1, we know for $C_{\mathrm{m}} \rightarrow 0$ that a Hopf branch starts at $I_{\mathrm{Hopf}}$ and that this branch does not return to the limit $C_{\mathrm{m}} \rightarrow 0$. Because limit-cycle bifurcation branches cannot cross each other in a planar system, the Hopf branch can furthermore not cross the big HOM branch. Instead, it connects to the fold bifurcation branch in a BT bifurcation at some point $\left(I_{\mathrm{SN} 1}, C_{\mathrm{BT} 1}\right)$. The ordering $I_{\mathrm{big} \mathrm{HOM}}<I_{\mathrm{Hopf}}$ from Observation 1 immediately implies an ordering in $C_{\mathrm{m}}$, i.e., $C_{\mathrm{BT} 1}<C_{\text {big SNL}}$. In summary, we have shown in this lemma that $C_{\mathrm{BT} 1}<C_{\text {big SNL }}<C_{\mathrm{SNIC}}$.

These arguments have proven the bifurcation sequence in the lower part of the bifurcation diagram arising from the limit $C_{\mathrm{m}} \rightarrow 0$. In the following, we use the unfolding of a second BT point to show the upper part of the bifurcation diagram.

\section{The upper part of the bifurcation diagram, $C_{\mathrm{m}}>C_{\mathrm{SNIC}}$}

Observation 3: The bifurcation diagram contains exactly two BT points. Kirst et al. identified the BT point for a generic class of conductance-based neuron models (including our model group) at a capacitance value that can be calculated from the input current at which the fold bifurcation occurs (Ref. [27], Supplemental Material). With the twofold bifurcation branches occurring in our model group at input currents $I_{\mathrm{SN} 0}$ and $I_{\mathrm{SN} 1}$, we find one unique BT point on each fold branch. Lemma 3 identified one of them at the $\mathrm{BT}$ point $\left(I_{\mathrm{SN} 1}, C_{\mathrm{BT} 1}\right)$, and the second BT bifurcation occurs at some point $\left(I_{\mathrm{SN} 0}, C_{\mathrm{BT} 0}\right)$. From the BT point at $\left(I_{\mathrm{SN} 0}, C_{\mathrm{BT} 0}\right)$ arises by normal form theory a Hopf bifurcation branch and a branch of a small HOM bifurcation. Both depart in the direction of increasing input $I_{\mathrm{DC}}$, which will be used as before to constrain their location.

Observation 4: The second BT point lies in the upper part of the bifurcation diagram-The BT point at $\left(I_{\mathrm{SN} 0}, C_{\mathrm{BT} 0}\right)$ occurs at $C_{\mathrm{BTO}}>C_{\mathrm{SNIC}}$. We restrict the region accessible to the Hopf branch that arises from the BT point at $\left(I_{\mathrm{SN} 0}, C_{\mathrm{BTO}}\right)$ : A limit cycle bifurcation branch cannot cross other limit cycle bifurcation branches (in a planar system), and hence the Hopf branch cannot pass the SNIC bifurcation line between $\left(I_{\mathrm{SN} 1}, C_{\mathrm{SNIC}}\right)$ and $\left(I_{\mathrm{SN} 1}, C_{\text {big SNL }}\right)$ or the big HOM branch. Furthermore, (A2) demands that no stable fixed point exists for $I_{\mathrm{DC}}<I_{\mathrm{SN} 1}$ for $C_{\mathrm{m}}=C_{\mathrm{SNIC}}$, effectively preventing the Hopf branch to pass this line. The Hopf branch lies, hence, either entirely within or outside of the region bounded by these lines.

We show that the Hopf branch lies outside of this region by identifying this branch with the excitation block occurring at $C_{\mathrm{m}}=C_{\mathrm{SNIC}}$ : (A2) demands that the excitation block at some $I_{\mathrm{DC}}>I_{\mathrm{SN} 1}$, i.e., outside of the identified region. Around the excitation block, $P_{\text {block }}$ is stabilized by a Hopf bifurcation. This Hopf bifurcation affects $P_{\text {block }}$ and hence belongs to the same branch of Hopf bifurcations that arises at the BT point at ( $\left.I_{\mathrm{SN} 0}, C_{\mathrm{BT} 0}\right)$, because this is where $P_{\text {block }}$ is created. With that, the Hopf branch must lie outside the region denoted above and correspondingly also the BT point at its end. We hence conclude $C_{\mathrm{BTO}}>C_{\mathrm{SNIC}}$.

Lemma 4: The small SNL bifurcation-A second $S N L$ bifurcation occurs at some $C_{\text {small SNL }}>C_{\text {SNIC }}$. The branch of the small HOM bifurcation that arises from the BT point at $\left(I_{\mathrm{SN} 0}, C_{\mathrm{BT} 0}\right)$ (see Observation 3 ) continues by Lemma 1 in the direction of increasing input $I_{\mathrm{DC}}$. Hence, we find some $C_{\mathrm{m}}=C_{\text {small SNL }}$ for which the small HOM branch connects to the fold bifurcation at $I_{\mathrm{SN} 1}$. At the connection point, the HOM branch must be stable by Lemma 2 . We identify the point $\left(I_{\mathrm{SN} 1}, C_{\text {small SNL }}\right)$ as small SNL bifurcation.

For the overall proof, it remains to show the ordering $C_{\text {small SNL }}>C_{\text {SNIC }}$. For that, we observe that a limit cycle exists between the small HOM and the Hopf branch arising from the BT point and contrast this with the limit cycle arising from the SNIC bifurcation. As the Hopf bifurcation has to terminate the limit cycle of the SNIC bifurcation at $C_{\text {SNIC }}$ [following (A2)], it cannot terminate the limit cycle arising from the small HOM bifurcation at this capacitance value. This leaves only the possibility for the SNL point to occur at some $C_{\text {small SNL }}>C_{\text {SNIC }}$.

In summary, we have shown that $C_{\mathrm{BT} 1}<C_{\text {big SNL }}<$ $C_{\text {SNIC }}<C_{\text {small SNL. }}$ This generic bifurcation structure occurs with the membrane capacitance $C_{\mathrm{m}}$ as bifurcation parameter at $I_{\mathrm{DC}}=I_{\mathrm{SN} 1}$. For a model starting at a SNIC bifurcation, a variation in the capacitance will thus pass an SNL bifurcation before a BT point is reached.
[1] L. L. Gollo and M. Breakspear, Philos. Trans. R. Soc. London, Ser. B 369, 20130532 (2014).

[2] P. Villegas, P. Moretti, and M. A. Muñoz, Sci. Rep. 4, 5990 (2014).

[3] Y. D. Sato and K. Aihara, Neural Comput. 26, 2395 (2014).

[4] A. L. Barth and J. F. A. Poulet, Trends Neurosci. 35, 345 (2012).

[5] F. C. Hoppensteadt and E. M. Izhikevich, Weakly Connected Neural Networks (Springer Science \& Business Media, Berlin, 1997).

[6] W. Gerstner and W. M. Kistler, Spiking Neuron Models: Single Neurons, Populations, Plasticity (Cambridge University Press, Cambridge, UK, 2002).

[7] P. Dayan and L. F. Abbott, Theoretical Neuroscience: Computational and Mathematical Modeling of Neural Systems
(Massachusetts Institute of Technology Press, Cambridge, MA, 2001).

[8] R. D. Traub, R. K. Wong, R. Miles, and H. Michelson, J. Neurophysiol. 66, 635 (1991).

[9] X.-J. Wang and G. Buzsáki, J. Neurosci. 16, 6402 (1996).

[10] A. L. Hodgkin, J. Physiol. 107, 165 (1948).

[11] G. B. Ermentrout and N. Kopell, SIAM J. Appl. Math. 46, 233 (1986).

[12] J. Rinzel and G. B. Ermentrout, Analysis of neural excitability and oscillations, in Methods in Neuronal Modeling, 2nd ed., edited by C. Koch and I. Segev (MIT Press, Cambridge, MA, 1998), pp. 251-291.

[13] P. Arhem, G. Klement, and C. Blomberg, Biophys. J. 90, 4392 (2006). 
[14] K. M. Stiefel, B. S. Gutkin, and T. J. Sejnowski, J. Comput. Neurosci. 26, 289 (2009).

[15] S. A. Prescott, S. Ratté, Y. D. Koninck, and T. J. Sejnowski, J. Neurophysiol. 100, 3030 (2008).

[16] E. Phoka, H. Cuntz, A. Roth, and M. Häusser, PLoS Comput. Biol. 6, e1000768 (2010).

[17] M. J. E. Richardson, N. Brunel, and V. Hakim, J. Neurophysiol. 89, 2538 (2003).

[18] S. Schreiber, I. Samengo, and A. V. M. Herz, J. Neurophysiol. 101, 2239 (2009).

[19] S. Schreiber, I. Erchova, U. Heinemann, and A. V. M. Herz, J. Neurophysiol. 92, 408 (2004).

[20] E. Izhikevich, Int. J. Bifurcation Chaos 10, 1171 (2000).

[21] S. Schecter, SIAM J. Math. Anal. 18, 1142 (1987).

[22] The SNL bifurcation [21] is also known as saddle-node homoclinic orbit, saddle-node noncentral homoclinic, saddle-node separatrix-loop bifurcation [23], or orbit flip bifurcation [24].

[23] E. M. Izhikevich, Dynamical Systems in Neuroscience (MIT Press, Cambridge, MA, 2007).

[24] A. J. Homburg and B. Sandstede, in Handbook of Dynamical Systems III, edited by H. W. Broer, F. Takens, and B. Hasselblatt (Elsevier, Amsterdam, 2010).

[25] P. Jiruska, M. de Curtis, J. G. R. Jefferys, C. A. Schevon, S. J. Schiff, and K. Schindler, J. Physiol. 591, 787 (2013).

[26] S. J. Schiff, Philos. Trans. R. Soc. London, Ser. A 368, 2269 (2010).

[27] C. Kirst, J. Ammer, F. Felmy, A. Herz, and M. Stemmler, bioRxiv (2015), doi:10.1101/022475.

[28] U. Pereira, P. Coullet, and E. Tirapegui, Entropy 17, 7859 (2015).

[29] A. A. Lazar, IEEE Trans. Inf. Theory 56, 821 (2010).

[30] A. A. Lazar and Y. B. Slutskiy, Neural Comp. 26, 264 (2014).

[31] Y. A. Kuznetsov, Elements of Applied Bifurcation Theory (Springer Science \& Business Media, Berlin, 2013).

[32] B. Ermentrout, Neural Comput. 8, 979 (1996).

[33] E. Brown, J. Moehlis, and P. Holmes, Neural Comput. 16, 673 (2004).

[34] K. M. Shaw, Y.-M. Park, H. J. Chiel, and P. J. Thomas, SIAM J. Appl. Dyn. Syst. 11, 350 (2012).

[35] A. Pikovsky, M. Rosenblum, and J. Kurths, Synchronization: A Universal Concept in Nonlinear Sciences (Cambridge University Press, Cambridge, UK, 2003).

[36] Y. Kuramoto, Chemical Oscillations, Waves, and Turbulence (Springer Science \& Business Media, Berlin, 1984).

[37] G. B. Ermentrout and D. H. Terman, Mathematical Foundations of Neuroscience (Springer Science \& Business Media, Berlin, 2010).

[38] H. Daido, Prog. Theor. Phys. 88, 1213 (1992).

[39] H. Daido, Phys. D (Amsterdam, Neth.) 91, 24 (1996).

[40] J.-H. Schleimer and M. Stemmler, Phys. Rev. Lett. 103, 248105 (2009).

[41] A. Franci, G. Drion, V. Seutin, and R. Sepulchre, PLoS Comput. Biol. 9, e1003040 (2013).

[42] P. De Maesschalck and M. Wechselberger, J. Math. Neurosci. 5, 16 (2015).

[43] S. Blankenburg, W. Wu, B. Lindner, and S. Schreiber, J. Comput. Neurosci. 39, 349 (2015).

[44] C. C. Chow and J. A. White, Biophys. J. 71, 3013 (1996).

[45] S. Hong, B. Agüera y Arcas, and A. L. Fairhall, Neural Comput. 19, 3133 (2007).

[46] A. L. Hodgkin and A. F. Huxley, J. Physiol. 117, 500 (1952).
[47] The bifurcation structure of the HH model arises in our model when the twofold bifurcations collide in a cusp bifurcation. This can be achieved by parameters that affect the shape of the nullclines, but is not possible with input and capacitance as bifurcation parameters as used here.

[48] The normal form used to calculate PRCs for the generalized Hopf bifurcation in Ref. [33] assumes a circular symmetric fold of limit cycles bifurcation. This holds locally around the subcritical Hopf bifurcation, but is unrealistic for full-blown pulselike spikes, for which a separation of time scales is required in the dynamics.

[49] Y. Tsubo, J.-n. Teramae, and T. Fukai, Phys. Rev. Lett. 99, 228101 (2007)

[50] A. Abouzeid and B. Ermentrout, Phys. Rev. E 84, 061914 (2011).

[51] J. Hesse and S. Schreiber, Curr. Biol. 25, R324 (2015).

[52] K. Bozek, Y. Wei, Z. Yan, X. Liu, J. Xiong, M. Sugimoto, M. Tomita, S. Pbo, C. C. Sherwood, P. R. Hof, J. J. Ely, Y. Li, D. Steinhauser, L. Willmitzer, P. Giavalisco, and P. Khaitovich, Neuron 85, 695 (2015).

[53] G. Eyal, M. B. Verhoog, G. Testa-Silva, Y. Deitcher, J. C. Lodder, R. Benavides-Piccione, J. Morales, J. DeFelipe, C. P. de Kock, H. D. Mansvelder et al., eLife 5, e16553 (2016).

[54] T. H. Rumbell, D. Dragulji, A. Yadav, P. R. Hof, J. I. Luebke, and C. M. Weaver, J. Comput. Neurosci. 41, 65 (2016).

[55] G. Hadjilambreva, E. Mix, A. Rolfs, J. Müller, and U. Strauss, J. Neurophysiol. 93, 843 (2005).

[56] C. P. Billimoria, R. A. DiCaprio, J. T. Birmingham, L. F. Abbott, and E. Marder, J. Neurosci. 26, 5910 (2006).

[57] K. M. Stiefel, B. S. Gutkin, and T. J. Sejnowski, PLoS ONE 3, e3947 (2008).

[58] T. Netoff, M. A. Schwemmer, and T. J. Lewis, Phase Response Curves in Neuroscience (Springer, New York, 2012), pp. 95-130.

[59] S. Wang, M. M. Musharoff, C. C. Canavier, and S. Gasparini, J. Neurophysiol. 109, 2757 (2013).

[60] B. S. Gutkin, G. B. Ermentrout, and A. D. Reyes, J. Neurophysiol. 94, 1623 (2005).

[61] G. B. Ermentrout, B. Beverlin, II, T. Troyer, and T. I. Netoff, J. Comput. Neurosci. 31, 185 (2011).

[62] The deposition of energy by infrared laser pulses leads to a fast increase in the membrane capacitance $[63,64]$ changing the capacitive current $I_{\text {cap }}=\frac{d C_{\mathrm{m}} v}{d t}=\dot{C}_{\mathrm{m}} v+C_{\mathrm{m}} \dot{v}$. Given the time course of energy dissipation, the fast transient capacitance increase, sufficient to excite the neuron [64], is followed by a time interval of increased $C_{\mathrm{m}}$. Our study applies to neuronal properties beyond the initial transient.

[63] J. Wells, C. Kao, K. Mariappan, J. Albea, E. D. Jansen, P. Konrad, and A. Mahadevan-Jansen, Opt. Lett. 30, 504 (2005).

[64] M. G. Shapiro, K. Homma, S. Villarreal, C.-P. Richter, and F. Bezanilla, Nat. Commun. 3, 736 (2012).

[65] Z. Levnajić, Sci. Rep. 2, 967 (2012).

[66] Actually, in some theories, single-cell characteristics fade into the background, while network features determine the macroscopic states $[2,67,68]$. Moreover, it has been concluded that cell heterogeneity in deterministic parameters can be approximated by white noise $[69,70]$. This, however, is due only to the fact that the varied parameters were ineffective in altering the neurons' computational and synchronization properties. For neurons close to an SNL point these results will not hold. 
[67] D. J. Amit and N. Brunel, Network 8, 373 (1997).

[68] N. Brunel and V. Hakim, Neural Comput. 11, 1621 (1999).

[69] A. K. Alijani and M. J. E. Richardson, Phys. Rev. E 84, 011919 (2011).

[70] J. F. Mejias and A. Longtin, Phys. Rev. Lett. 108, 228102 (2012).

[71] D. Witthaut and M. Timme, New J. Phys. 14, 083036 (2012).

[72] S. A. Marvel, S. H. Strogatz, and J. M. Kleinberg, Phys. Rev. Lett. 103, 198701 (2009).

[73] M. C. Eguia and G. B. Mindlin, Phys. Rev. E 60, 1551 (1999).

[74] J. F. Martinez Avila, H. L. D. de S. Cavalcante, and J. R. Rios Leite, Phys. Rev. Lett. 93, 144101 (2004).

[75] Y. Ekşioğlu, Ö. E. Müstecaplığlu, and K. Güven, Phys. Rev. A 87, 023823 (2013).

[76] R. Labouvie, B. Santra, S. Heun, and H. Ott, Phys. Rev. Lett. 116, 235302 (2016).
[77] N. Shimizu, T. Morooka, and M. Morisue, Jpn. J. Appl. Phys. 34, 5588 (1995).

[78] M. Romanelli, A. Thorette, M. Brunel, T. Erneux, and M. Vallet, Phys. Rev. A 94, 043820 (2016).

[79] F. W. Schneider and A. F. Münster, J. Phys. Chem. 95, 2130 (1991).

[80] V. Balakotaiah, S. M. S. Dommeti, and N. Gupta, Chaos 9, 13 (1999).

[81] E. J. Doedel and B. E. Oldeman, AUTO-07P: Continuation and Bifurcation Software for Ordinary Differential Equations (Concordia University, Montreal, Quebec, Canada, 2009).

[82] E. M. Izhikevich and F. C. Hoppensteadt, SIAM J. Appl. Math. 63, 1935 (2003).

[83] C. Chicone, Ordinary Differential Equations With Applications (Springer Science \& Business Media, Berlin, 2006). 